\title{
A Universal Optimal Consumption Rate for an Insider
}

\author{
Bernt Øksendal \\ CMA, Dept. of Mathematics, \\ University of Oslo, Box 1053, Blindern, N-0316 Oslo, Norway \\ and \\ Norwegian School of Economics and Business Administration, \\ Helleveien 30, N-5045 Bergen, Norway
}

$$
\begin{aligned}
& \text { Abstract } \\
& \text { We consider a cash flow } X^{(c)}(t) \text { modelled by the stochastic equation } \\
& \begin{array}{r}
d X^{(c)}(t)=X^{(c)}\left(t^{-}\right)\left[\mu(t) d t+\sigma(t) d^{-} B(t)\right. \\
\left.+\int_{\mathbb{R}} \theta(t, z) \tilde{N}\left(d^{-} t, d z\right)\right]-c(t) d t ; \quad t \geq 0
\end{array} \\
& X^{(c)}(0)=x>0
\end{aligned}
$$

where $B(\cdot)$ and $\tilde{N}(\cdot, \cdot)$ is a Brownian motion and a Poissonian random measure, respectively, and $c(t) \geq 0$ is the consumption/dividend rate. No assumptions are made on adaptedness of the coefficients $\mu, \sigma, \theta$ and $c$, and the (possibly anticipating) integrals are interpreted in the forward integral sense. We solve the problem to find the consumption rate $c(\cdot)$ which maximizes the expected discounted utility given by

$$
J(c)=E\left[\int_{0}^{\tau} e^{-\delta(t)} \ln c(t) d t+\gamma e^{-\delta(\tau)} \ln X^{(c)}(\tau)\right] .
$$

MSC (2000): 93E20, 60G51, 60G57, 60H05, 91B28, 91B76

Keywords: Optimal consumption/investment rate, Lévy process, insider trading, forward integrals 
Here $\delta(t) \geq 0$ is a given measurable stochastic process representing a discounting exponent and $\tau$ is a random time with values in $(0, \infty)$, representing a terminal/default time, while $\gamma \geq 0$ is a known constant.

\section{Introduction}

Suppose the cash flow at time $t$ is modelled by a geometric Brownian motion given by

$$
d X(t)=X(t)[\mu d t+\sigma d B(t)] ; \quad X(0)=x>0, \quad t \geq 0 .
$$

Here $B(t)=B(t, \omega)$ is a Brownian motion on a filtered probability space $\left(\Omega, \mathcal{F}, \mathcal{F}_{t}, P\right), \mu, \sigma$ and $x$ are known constants and the differential $d B(t)$ is interpreted in the Itô sense, i.e. (1.1) is just a shorthand notation for the Itô stochastic integral equation

$$
X(t)=x+\int_{0}^{t} \mu X(s) d s+\int_{0}^{t} \sigma X(s) d B(s) ; \quad t \geq 0 .
$$

Suppose that at any time $t$ we are free to take out consumption (or dividends) at a rate $c(t)=c(t, \omega) \geq 0$. The corresponding cash flow equation is then

$$
d X^{(c)}(t)=X^{(c)}[\mu d t+\sigma d B(t)]-c(t) d t ; \quad X^{(c)}(0)=x>0
$$

In the classical setup it is assumed that $c(t)$ is adapted with respect to the filtration $\mathcal{F}_{t}$ generated by $B(s) ; s \leq t$. This ensures that (1.3) still makes sense as an Itô stochastic differential equation. Moreover, this is a natural assumption from a modelling point of view: The decision about the cosumption rate at time $t$ should only depend upon the information obtained by observing the cash amount $X(s) ; s \leq t$ and not upon any future values of $X(r)$.

Now assume that the consumer has a logarithmic utility of her consumption rate. The expected total discounted utility of a chosen consumption rate $c(\cdot)$ is then

$$
J(c)=E\left[\int_{0}^{\infty} e^{-\delta t} \log c(t) d t\right]
$$


where $\delta>0$ (constant) is a given discounting exponent. Let the set $\mathcal{A}$ of admissible controls (consumption rates) be the set of $\mathcal{F}_{t}$-adapted $c(t) \geq 0$ such that $X^{(c)}(t) \geq 0$ for all $t$. Consider the following problem:

Problem 1.1 Find $c^{*} \in \mathcal{A}$ such that

$$
J\left(c^{*}\right)=\sup _{c \in \mathcal{A}} J(c)
$$

Such a control $c^{*}$ (if it exists) is called an optimal control for Problem 1.1.

It is well-known, and easy to prove by using stochastic control theory, that the optimal control $c^{*}$ for Problem 1.1 is given by (in feedback form)

$$
c^{*}(t)=\delta X^{\left(c^{*}\right)}(t) .
$$

In other words, it is optimal to consume at a rate proportional to current cash amount, with a constant of proportionality equal to the discounting exponent.

This is a remarkably simple result. Note in particular that $c^{*}$ does not depend on the parameters $\mu$ and $\sigma$. It is natural to ask if this result remains valid in a more general setting. More precisely, we ask what happens if we allow the following generalizations:

(i) We add a jump term (represented by a Poissonian random measure) in (1.3).

(ii) We replace the constant coefficients by measurable stochastic processes: $\delta=\delta(t, \omega), \mu=\mu(t, \omega)$ etc.

(iii) We do not assume that these coefficient processes are $\mathcal{F}_{t}$-adapted, but we allow them to be arbitrary $\mathcal{F}_{\infty}$-measurable processes.

(iv) We introduce a stochastic terminal time (or default time) $\tau$, with values in $[0, \infty]$. This random time is not necessarily an $\mathcal{F}_{t}$-stopping time, but just assumed to be $\mathcal{F}_{\infty}$-measurable.

(v) We assume that the consumption rate $c(t)$ is adapted to some filtration $\mathcal{H}_{t}$, without any prior assumption about the relation betweeen $\mathcal{F}_{t}$ and $\mathcal{H}_{t}$. 
Two special cases in (v) are:

$$
\mathcal{H}_{t} \subset \mathcal{F}_{t} \text { for all } t \geq 0
$$

In this case the consumer has less information than what is represented by the filtration of the underlying driving process(es). This is often called a partial observation control problem. We refer to [B] for more information about this topic.

$$
\mathcal{F}_{t} \subset \mathcal{H}_{t} \text { for all } t \geq 0
$$

In this case the consumer has more information than what can be obtained by observing the driving processes. Such information is called inside information and in this case the consumer is called an insider. For example, in the original model (1.3) the extra information available could be the future value $B(T)$ of the underlying Brownian motion at some time $T>0$.

In the cases (iii), (iv) and (v2) it is clear that the corresponding equation for the cash flow $X^{(c)}(t)$ is no longer an Itô stochastic differential equation, because of the anticipating coefficients, the default time and the control. So we must explain the meaning of stochastic integrals of the form

$$
\int_{0}^{t} \varphi(s, \omega) d B(s)
$$

when $\varphi(s, \omega)=\varphi(s)=X^{(c)}(s) \sigma(s)$ is not $\mathcal{F}_{s}$-adapted. We choose to model (1.7) by using forward integrals, which are defined as follows

Definition 1.2 ([RV1]) The forward integral of a stochastic process $\psi(t)$ (not necessarily $\mathcal{F}_{t}$-adapted) with respect to $B(t)$ is defined by

$$
\int_{0}^{\infty} \psi(t) d^{-} B(t):=\lim _{\epsilon \rightarrow 0} \int_{0}^{\infty} \psi(t) \frac{B(t+\epsilon)-B(t)}{\epsilon} d t
$$

if the limit exists in probability.

We refer to [RV1] for more information about forward integrals.

Two reasons for modeling the anticipating situation above by using forward integrals are 
(a) If the process $\psi(t)$ is forward integrable and caglad (i.e. left continuous with right limits) then, for all $T>0$,

$$
\int_{0}^{T} \psi(t) d^{-} B(t)=\lim _{\Delta t_{j} \rightarrow 0} \sum_{j} \psi\left(t_{j}\right) \Delta B\left(t_{j}\right)
$$

where $0=t_{0}<t_{1}<\cdots<t_{N}=T$ is a partition of $[0, T], \Delta t_{j}=t_{j+1}-t_{j}$ and $\Delta B\left(t_{j}\right)=B\left(t_{j+1}\right)-B\left(t_{j}\right)$. So the forward integral is nothing but a special case of a Riemann integral, where the value of the integrand, $\psi\left(t_{j}\right)$, is always chosen to be the value at the left end point of the subinterval $\left[t_{j}, t_{j+1}\right]$. This makes it a natural model for the growth of wealth in finance, where the integrand typically represents the portfolio chosen and the integrator typically represents the stock price.

(b) Secondly, if the integrand $\psi(t)$ is adapted to a filtration $\mathcal{G}_{t}$ and $B(t)$ is a semimartingale with respect to $\mathcal{G}_{t}$, then the forward integral of $\psi$ coincides with the (extended) Itô integral in the semimartingale setting.

We refer to e.g. $[\mathrm{B} \varnothing 1]$ and $[\mathrm{B} \varnothing 2]$ for more information about (a) and (b) above.

Recently there has been an increased interest in finance models which also include jumps. See e.g. [S] and the references therein. Such models can be represented in terms of Lévy processes. They are defined as

Definition 1.3 A (1-dimensional) Lévy process is an $\mathcal{F}_{t}$-adapted process $\eta(t, \omega):[0, \infty) \times \Omega \rightarrow \mathbb{R}$ (where as before $\left(\Omega, \mathcal{F}, \mathcal{F}_{t}, P\right)$ is a filtered probability space) with stationary, independent increments and cadlag paths (right continuous with left limits) paths. We also assume that

$$
\eta(0)=0 .
$$

By the Lévy decomposition theorem every Lévy process $\eta$ such that $E\left[\eta^{2}(t)\right]<\infty$ for all $t$ can be written on the form

$$
\eta(t)=a t+b B(t)+\int_{0}^{t} \int_{\mathbb{R}} z \tilde{N}(d s, d z),
$$


where

$$
\tilde{N}(d s, d z):=N(d s, d z)-d s \nu(d z)
$$

is the compensated Poisson random (jump) measure of $\eta(\cdot)$. Here $N(d s, d z)$ is the jump measure of $\eta(\cdot)$ defined by, for $t>0$ and $Z \subset \mathbb{R} \backslash\{0\}$,

$$
\begin{aligned}
N((0, t), Z)= & \text { the number of jumps } \Delta \eta(s):=\eta(s)-\eta\left(s^{-}\right) \in Z \\
& \text { of } \eta(s) \text { of size } \Delta \eta(s) \in Z \text { up to time } t,
\end{aligned}
$$

and finally

$$
\nu(Z):=E[N([0,1], Z)] ; \quad Z \subset \mathbb{R} \backslash\{0\}
$$

is the Lévy measure of $\eta(\cdot)$.

An integration theory with Lévy processes $\eta(\cdot)$ as integrators was developed already by Itô $[\mathrm{I}]$. See $[\mathrm{P}]$ for an account of this. It turns out that the theory can be extended to forward integrals with respect to $\eta(\cdot)$ and - more generally - with respect to the Poisson random measure $\tilde{N}(\cdot, \cdot)$, as follows:

Definition 1.4 [DMØP1] The forward integral

$$
J(\theta)=\int_{0}^{T} \int_{\mathbb{R}} \theta(t, z) \tilde{N}\left(d^{-} t, d z\right)
$$

of a caglad stochastic function $\theta(t, z)=\theta(t, z, \omega)$ is defined by

$$
J(\theta)=\lim _{m \rightarrow \infty} \int_{0}^{T} \int_{U_{m}} \theta(t, z) \tilde{N}(d t, d z)
$$

if the limit exists in $L^{2}(P)$. Here $U_{m} ; m=1,2, \ldots$ is an increasing sequence of compact sets with $\nu\left(U_{m}\right)<\infty$ for all $m$ and $\bigcup_{m=1}^{\infty} U_{m}=\mathbb{R} \backslash\{0\}$.

There is an Itô formula for forward integrals. This was first proved in [RV2] in the Brownian motion case $(\tilde{N}=0)$ and then extended to the general case in $[\mathrm{DM} \varnothing \mathrm{P} 1]$. The result is the following: 
Theorem 1.5 [DMØP1] (Itô formula for forward integrals with respect to Lévy processes)

Let

$$
d^{-} X(t)=\alpha(t) d t+\beta(t) d^{-} B(t)+\int_{\mathbb{R}} \theta(t, z) \tilde{N}\left(d^{-} t, d z\right)
$$

be a forward process with respect to $B(\cdot)$ and $\tilde{N}(\cdot, \cdot)$, where $\alpha(\cdot), \beta(\cdot)$ and $\theta(\cdot, \cdot)$ are measurable processes such that $\beta$ and $\theta$ are forward integrable and

$$
\int_{0}^{t}\left(\alpha^{2}(s)+\beta^{2}(s)+\int_{\mathbb{R}} \theta^{2}(s, z) \nu(d z)\right) d s<\infty \quad \text { for all } t,
$$

and assume that $\theta(t, z)$ is locally bounded in $z$ near $z=0$ for a.a. $t, \omega$.

Let $f \in C^{2}(\mathbb{R})$ and define

$$
Y(t)=f(X(t)) .
$$

Then $Y(t)$ is also a forward process and

$$
\begin{aligned}
& d^{-} Y(t)=f^{\prime}(X(t))\left[\alpha(t) d t+\beta(t) d^{-} B(t)\right]+\frac{1}{2} f^{\prime \prime}(X(t)) \beta^{2}(t) d t \\
& \quad+\int_{\mathbb{R}}\left\{f\left(X\left(t^{-}\right)+\theta(t, z)\right)-f\left(X\left(t^{-}\right)\right)-f^{\prime}\left(X\left(t^{-}\right)\right) \theta(t, z)\right\} \nu(d z) d t \\
& \quad+\int_{\mathbb{R}}\left\{f\left(X\left(t^{-}\right)+\theta(t, z)\right)-f\left(X\left(t^{-}\right)\right)\right\} \tilde{N}\left(d^{-} t, d z\right) .
\end{aligned}
$$

We now apply this forward stochastic calculus machinery to study the extension of Problem 1.1 given in (i)-(v) above.

\section{The general problem}

We now consider a cash flow which, after being subject to a consumption/dividend rate $c(t) \geq 0$ is modelled by the equation

$$
\begin{aligned}
& d X^{(c)}(t)=X^{(c)}\left(t^{-}\right)\left[\mu(t) d t+\sigma(t) d^{-} B(t)+\int_{\mathbb{R}} \theta(t, z) \tilde{N}\left(d^{-} t, d z\right)\right] \\
& \quad-c(t) d t ; \quad t \geq 0 \\
& X^{(c)}=x>0 .
\end{aligned}
$$


Here $B(t)$ and $\eta(t)=\int_{0}^{t} \int_{\mathbb{R}} z \tilde{N}(d s, d z)$ is a Brownian motion and an (independent) pure jump Lévy process, respectively, on a filtered probability space $\left(\Omega, \mathcal{F},\left\{\mathcal{F}_{t}\right\}_{t \geq 0}, P\right)$. As before we let $\nu$ denote the Lévy measure of $\eta$ and we let $\tilde{N}(\cdot, \cdot)$ denote the compensated Poisson random measure of $\eta(\cdot)$, so that

$$
\tilde{N}(d t, d z)=N(d t, d z)-\nu(d z) d t
$$

$N(\cdot, \cdot)$ being the Poisson random measure of $\eta(\cdot)$.

Let $\left\{\mathcal{H}_{t}\right\}_{t \geq 0}$ be another filtration, with no a priori relation to $\left\{\mathcal{F}_{t}\right\}_{t \geq 0}$. The $\sigma$-algebra $\mathcal{H}_{t}$ represents the information available to the agent at time $t$. We make no a priori adaptedness conditions on the given processes $\mu(t), \sigma(t)$ and $\theta(t, z)$, except that they are $\mathcal{F}_{\infty}$-measurable for all $t, z$. This allows us to model the situation where the cash flow may be influenced by the actions of other traders who are insiders.

The corresponding stochastic differential equation (2.1) is interpreted as the forward stochastic integral equation

$$
\begin{aligned}
X^{(c)}(t)= & x+\int_{0}^{t} X^{(c)}(s) \mu(s) d s+\int_{0}^{t} X^{(c)}(s) \sigma(s) d^{-} B(s) \\
& +\int_{0}^{t} \int_{\mathbb{R}} X^{(c)}\left(s^{-}\right) \theta(s, z) \tilde{N}\left(d^{-} s, d z\right)-\int_{0}^{t} c(s) d s ; \quad t \geq 0,
\end{aligned}
$$

where $d^{-} B(s)$ and $\tilde{N}\left(d^{-} s, d z\right)$ indicate that forward integral interpretation is being used (see Section 1).

Let $\delta(t) \geq 0$ be a given $\mathcal{F}_{\infty}$-measurable process, modelling a discounting exponent, and let $\tau: \Omega \rightarrow[0, \infty]$ be a given $\mathcal{F}_{\infty}$-measurable random variable, representing a terminal or default time for the cash flow. We consider the problem to maximize the sum of the expected total discounted logarithmic utility of the consumption rate $c(t)$ up to the default time $\tau$ and the logarithmic utility of the terminal cash amount $X^{(c)}(\tau)$, given by

$$
J(c)=E\left[\int_{0}^{\tau} e^{-\delta(t)} \ln c(t) d t+\gamma e^{-\delta(\tau)} \ln X^{(c)}(\tau)\right],
$$

subject to the condition that $X^{(c)}(t)>0$ for all $t<\tau$. Here $\gamma \geq 0$ is a constant and $E$ denotes the expectation with respect to the probability law 
$P$ of $(B(\cdot), \eta(\cdot))$. We assume that the choice of $c(\cdot)$ has no influence on $\delta, \mu, \sigma, \theta$ or $\tau$.

In the next section we show that, under some conditions, the optimal consumption/dividend rate $c^{*}(t)$ for (2.3) has the simple feedback form

$$
c^{*}(t)=\lambda^{*}(t) X^{\left(c^{*}\right)}(t)
$$

where

$$
\lambda^{*}(t)=\frac{E\left[\mathcal{X}_{[0, \tau]}(t) e^{-\delta(t)} \mid \mathcal{H}_{t}\right]}{E\left[\mathcal{X}_{[0, \tau]}(t)\left(\int_{t}^{\tau} e^{-\delta(s)} d s+\gamma e^{-\delta(\tau)}\right) \mid \mathcal{H}_{t}\right]}
$$

See Theorem 3.3.

Note that $\lambda^{*}(t)$ does not depend on any of the coefficients $\mu(\cdot), \sigma(\cdot)$ and $\theta(\cdot)$.

The problem discussed in this paper is related to the optimal consumption and portfolio problems associated with a random time horizon studied in $[\mathrm{BEJM}],[\mathrm{BP}]$ and $[\mathrm{EJ}]$. However, our approach is different.

\section{The main result}

In this section we give a precise statement and a proof of the main result of this paper.

We assume the following about the market:

$$
\mu(t), \sigma(t), \theta(t, z), \delta(t) \quad \text { and } \quad \tau: \Omega \rightarrow[0, \infty]
$$

are $\mathcal{F}_{\infty}$-measurable for all $t, z$

(3.2) $\quad \theta(t, z)>-1 \quad$ for a.a. $t, z$ with respect to $d t \times \nu(d z)$

(3.3) $\sigma(s)$ and $\theta(s, z)$ are caglad (with respect to $s$ )

and the forward integrals

$$
\int_{0}^{\tau} \sigma(s) d^{-} B(s) \text { and } \int_{0}^{\tau} \int_{\mathbb{R}} \ln (1+\theta(s, z)) \tilde{N}\left(d^{-} s, d z\right)
$$

exist and belong to $L^{1}(P)$

$$
E\left[\int_{0}^{\tau} e^{-\delta(t)} k(t) d t+e^{-\delta(\tau)} k(\tau)\right]<\infty, \quad \text { where }
$$




$$
\begin{aligned}
k(t) & =\int_{0}^{t}\left\{|\mu(s)|+\sigma^{2}(s)+\int_{\mathbb{R}}|\ln (1+\theta(s, z))-\theta(s, z)| \nu(d z)\right\} d s \\
+ & \left|\int_{0}^{t} \sigma(s) d^{-} B(s)\right|+\left|\int_{0} \int_{\mathbb{R}} \ln (1+\theta(s, z)) \tilde{N}\left(d^{-} s, d z\right)\right| .
\end{aligned}
$$

We now represent the consumption rate $c(t)$ by its fraction $\lambda(t)$ of the total wealth, i.e. we put

$$
\lambda(t)=\frac{c(t)}{X^{(c)}(t)} .
$$

We call $\lambda(t)$ the relative consumption rate. If $X^{(c)}(\tau)=0$, we put $\lambda(\tau)=0$.

If $\int_{0}^{t} \lambda(s) d s<\infty$ a.s., for all $t<\tau$, then the solution $X(t)=X^{(\lambda)}(t)$ of the corresponding wealth equation is, by the Itô formula for forward integrals, given by

$$
\begin{aligned}
X^{(\lambda)}(t)= & x \exp \left[\int _ { 0 } ^ { t } \left\{\mu(s)-\lambda(s)-\frac{1}{2} \sigma^{2}(s)\right.\right. \\
& \left.+\int_{\mathbb{R}}(\ln (1+\theta(s, z))-\theta(s, z)) \nu(d z)\right\} d s \\
& \left.+\int_{0}^{t} \sigma(s) d^{-} B(s)+\int_{0}^{t} \int_{\mathbb{R}} \ln (1+\theta(s, z)) \tilde{N}\left(d^{-} s, d z\right)\right] .
\end{aligned}
$$

Definition 3.1 The set $\mathcal{A}$ of admissible controls is the set of $\mathcal{H}_{t}$-adapted processes $\lambda(t) \geq 0$ such that

$$
\int_{0}^{\tau} \lambda(s) d s<\infty \quad \text { a.s. }
$$

and

$$
E\left[\int_{0}^{\tau} e^{-\delta(t)}|\ln \lambda(t)| d t+e^{-\delta(\tau)}\left|\ln X^{(\lambda)}(\tau)\right|\right]<\infty
$$


To each $\lambda \in \mathcal{A}$ we associate the consumption/dividend rate

$$
c_{\lambda}(t):=\lambda(t) X^{(\lambda)}(t) .
$$

The problem we study can now be formulated as follows:

Problem 3.2 Find $\lambda^{*} \in \mathcal{A}$ such that

$$
J\left(c_{\lambda^{*}}\right)=\sup _{\lambda \in \mathcal{A}} J\left(c_{\lambda}\right)
$$

where

$$
J\left(c_{\lambda}\right)=E\left[\int_{0}^{\tau} e^{-\delta(t)} \ln c_{\lambda}(t) d t+\gamma e^{-\delta(\tau)} \ln X^{(\lambda)}(\tau)\right]
$$

Such $\lambda^{*}-$ if it exists - is called an optimal control for Problem 3.2.

We can now state and prove our main result:

Theorem 3.3 Define

$$
\hat{\lambda}(t)=\frac{E\left[\mathcal{X}_{[0, \tau]}(t) e^{-\delta(t)} \mid \mathcal{H}_{t}\right]}{E\left[\mathcal{X}_{[0, \tau]}(t)\left(\int_{t}^{\tau} e^{-\delta(s)} d s+\gamma e^{-\delta(\tau)}\right) \mid \mathcal{H}_{t}\right]} ; \quad t \geq 0
$$

If $\hat{\lambda} \in \mathcal{A}$ then $\hat{\lambda}=\lambda^{*}$ is the optimal control for Problem 3.2. If $\hat{\lambda} \notin \mathcal{A}$ then an optimal control does not exist.

Proof. Choose $\lambda \in \mathcal{A}$ and put $c(t)=\lambda(t) X^{(\lambda)}(t)$. Then

$$
\begin{aligned}
J(c)= & E\left[\int_{0}^{\tau} e^{-\delta(t)} \ln c(t) d t+\gamma e^{-\delta(\tau)} \ln X^{(\lambda)}(\tau)\right] \\
= & E\left[\int_{0}^{\tau} e^{-\delta(t)}\left(\ln \lambda(t)-\int_{0}^{t} \lambda(s) d s\right) d t-\gamma e^{-\delta(\tau)} \int_{0}^{\tau} \lambda(t) d t\right] \\
& +K, \quad \text { where } \\
K= & E\left[\int_{0}^{\tau} e^{-\delta(t)} h(t) d t+\gamma e^{-\delta(\tau)}(\ln x+h(\tau))\right],
\end{aligned}
$$


with

$$
\begin{aligned}
h(t)= & \int_{0}^{t}\left\{\mu(s)-\frac{1}{2} \sigma^{2}(s)+\int_{\mathbb{R}}(\ln (1+\theta(s, z))-\theta(s, z)) \nu(d z)\right\} d s \\
& +\int_{0}^{t} \sigma(s) d^{-} B(s)+\int_{0}^{t} \int_{\mathbb{R}} \ln (1+\theta(s, z)) \tilde{N}\left(d^{-} s, d z\right) .
\end{aligned}
$$

Note that $K$ does not depend on $\lambda$. Now, by the Fubini theorem,

$$
\int_{0}^{\tau} e^{-\delta(t)} \int_{0}^{t} \lambda(s) d s d t=\int_{0}^{\tau}\left(\int_{t}^{\tau} e^{-\delta(s)} d s\right) \lambda(t) d t .
$$

Substituting this into (3.10) we get

$$
\begin{aligned}
J(c)-K=E\left[\int_{0}^{\tau}\left\{e^{-\delta(t)} \ln \lambda(t)-\lambda(t) \int_{t}^{\tau} e^{-\delta(s)} d s-\gamma e^{-\delta(\tau)} \lambda(t)\right\} d t\right. \\
=E\left[\int_{0}^{\infty} \mathcal{X}_{[0, \tau]}(t)\left\{e^{-\delta(t)} \ln \lambda(t)-\lambda(t) \int_{t}^{\tau} e^{-\delta(s)} d s-\gamma e^{-\delta(\tau)} \lambda(t)\right\} d t\right] \\
=E\left[\int_{0}^{\infty} E\left[\mathcal{X}_{[0, \tau]}(t)\left\{e^{-\delta(t)} \ln \lambda(t)-\lambda(t) \int_{t}^{\tau} e^{-\delta(s)} d s-\gamma e^{-\delta(\tau)} \lambda(t)\right\} \mid \mathcal{H}_{t}\right] d t\right] \\
=E\left[\int _ { 0 } ^ { \infty } \left\{\ln \lambda(t) E\left[\mathcal{X}_{[0, \tau]}(t) e^{-\delta(t)} \mid \mathcal{H}_{t}\right]\right.\right. \\
\left.\left.-\lambda(t) E\left[\mathcal{X}_{[0, \tau]}(t)\left(\int_{t}^{\tau} e^{-\delta(s)} d s+\gamma e^{-\delta(\tau)}\right) \mid \mathcal{H}_{t}\right]\right\} d t\right] .
\end{aligned}
$$

We can maximize this pointwise, for each $t, \omega$ : The concave function

$$
\begin{aligned}
f(\lambda):= & \ln \lambda \cdot E\left[\mathcal{X}_{[0, \tau]}(t) e^{-\delta(t)} \mid \mathcal{H}_{t}\right] \\
& -\lambda E\left[\mathcal{X}_{[0, \tau]}(t)\left(\int_{t}^{\tau} e^{-\delta(s)} d s+\gamma e^{-\delta(\tau)}\right) \mid \mathcal{H}_{t}\right]
\end{aligned}
$$


is maximal when

$$
\begin{aligned}
0= & f^{\prime}(\lambda)=\lambda^{-1} E\left[\mathcal{X}_{[0, \tau]}(t) e^{-\delta(t)} \mid \mathcal{H}_{t}\right] \\
& -E\left[\mathcal{X}_{[0, \tau]}(t)\left(\int_{t}^{\tau} e^{-\delta(s)} d s+\gamma e^{-\delta(\tau)}\right) \mid \mathcal{H}_{t}\right]
\end{aligned}
$$

This gives the only possible optimal dividend candidate

$$
\hat{\lambda}(t)=\frac{E\left[\mathcal{X}_{[0, \tau]}(t) e^{-\delta(t)} \mid \mathcal{H}_{t}\right]}{E\left[\mathcal{X}_{[0, \tau]}(t)\left(\int_{t}^{\tau} e^{-\delta(s)} d s+\gamma e^{-\delta(\tau)}\right) \mid \mathcal{H}_{t}\right]} .
$$

In particular, we get the following extension of the result (1.6) above:

Corollary 3.4 Suppose $\tau=\infty, \gamma=0$ and $\delta(t)=\delta_{0} t$ for some constant $\delta_{0}>0$. Then the optimal relative consumption rate is

$$
\lambda^{*}(t)=\delta_{0} .
$$

\section{Optimal consumption and portfolio}

In this section we apply the result from Section 3 to study the problem of optimal comsumption and portfolio for a trader (possibly with inside information) in a market possibly influenced by other traders with inside information.

Suppose we have a financial market with the following two investment possibilities:

(i) a bond, with price $S_{0}(t)$ at time $t$ given by

$$
d S_{0}(t)=\rho(t) S_{0}(t) d t ; \quad S_{0}(0)=1
$$

(ii) a stock, with price $S_{1}(t)$ at time $t$ given by

$$
\begin{aligned}
d S_{1}(t) & =S_{1}\left(t^{-}\right)\left[\alpha(t) d t+\beta(t) d^{-} B(t)+\int_{\mathbb{R}} \xi(t, z) \tilde{N}\left(d^{-} t, d z\right)\right] \\
S_{1}(0) & >0
\end{aligned}
$$


In addition we assume as before that we are given a discounting exponent process $\delta(t) \geq 0$ and a default (or bankruptcy) time $\tau: \Omega \rightarrow[0, \infty]$.

We make the similar assumptions as we did for the cash flow in Section 3 , i.e. we assume the following:

$\rho(t), \alpha(t), \beta(t), \xi(t, z), \delta(t)$ and $\tau$ are $\mathcal{F}_{\infty}$-measurable for all $t, z$

$\xi(t, z)>-1$ for a.a. $t, z$ with respect to $d t \times \nu(d z)$

$\beta(s)$ and $\xi(s, z)$ are caglad (with respect to $s$ ) for all $z$

and the forward integrals

$$
\int_{0}^{\tau} \sigma(s) d^{-} B(s) \text { and } \int_{0}^{\tau} \int_{\mathbb{R}} \ln (1+\xi(s, z)) \tilde{N}\left(d^{-} s, d z\right)
$$

exist and belong to $L^{1}(P)$

Now suppose that a trader is free to choose at any time $t$ both the relative consumption rate $\lambda(t)=c(t) / X(t) \geq 0$ and the fraction $\pi(t)$ of the current total wealth $X(t)$ to be invested in the stocks. The wealth process $X(t)=$ $X^{(\lambda, \pi)}(t)$ corresponding to the consumption-portfolio pair $(\lambda, \pi)$ is given by

$$
\begin{aligned}
& d X(t)= \rho(t)(1-\pi(t)) X(t) d t-\lambda(t) X(t) d t \\
&+\pi(t) X\left(t^{-}\right)\left[\alpha(t) d t+\beta(t) d^{-} B(t)+\int_{\mathbb{R}} \xi(t, z) \tilde{N}\left(d^{-} t, d z\right)\right] \\
&= X\left(t^{-}\right)[\{\rho(t)+(\alpha(t)-\rho(t)) \pi(t)-\lambda(t)\} d t \\
&\left.+\pi(t) \beta(t) d^{-} B(t)+\int_{\mathbb{R}} \pi(t) \xi(t, z) \tilde{N}\left(d^{-} t, d z\right)\right], \\
& X(0)=x>0 .
\end{aligned}
$$

As in Section 3 we assume that the information available to the trader at time $t$ is represented by a filtration $\mathcal{H}_{t} ; t \geq 0$, with no a priori relation to $\mathcal{F}_{t}$; $t \geq 0$.

Note that for each given portfolio choice $\pi(t)$ the equation (4.6) has the same form as the cash flow equation (2.2), with

$$
\begin{aligned}
\mu(s) & =\rho(s)+(\alpha(s)-\rho(s)) \pi(s) \\
\sigma(s) & =\pi(s) \beta(s) \\
\theta(s, z) & =\pi(s) \xi(s, z)
\end{aligned}
$$


In view of this the following definition is natural:

Definition 4.1 We say that a consumption-portfolio pair $(\lambda, \pi)$ is admissible if

(4.10) $\quad \lambda(s)$ and $\pi(s)$ are $\mathcal{H}_{s}$-measurable; $s \geq 0$

(4.11) the processes $\mu(\cdot), \sigma(\cdot)$ and $\theta(\cdot, \cdot)$ defined by (4.7)-(4.9)

satisfy conditions (3.2), (3.3) and (3.4)

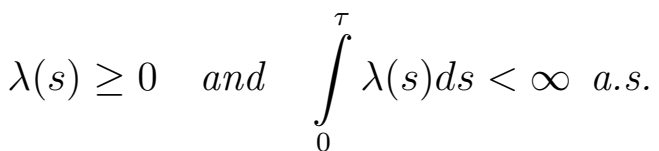

$$
E\left[\int_{0}^{\tau} e^{-\delta(t)} \mid \ln \left(\lambda(t) X^{(\lambda, \pi)}(t)\left|d t+\gamma e^{-\delta(\tau)}\right| \ln X^{(\lambda, \pi)}(\tau) \mid\right]<\infty .\right.
$$

The set of all admissible pairs $(\lambda, \pi)$ is denoted by $\mathcal{A}$.

We now consider the following problem:

Problem 4.2 Find $\left(\lambda^{*}, \pi^{*}\right) \in \mathcal{A}$ such that

$$
J\left(\lambda^{*}, \pi^{*}\right)=\sup _{(\lambda, \pi) \in \mathcal{A}} J(\lambda, \pi),
$$

where

$$
J(\lambda, \pi)=E\left[\int_{0}^{\tau} e^{-\delta(t)} \ln \left(\lambda(t) X^{(\lambda, \pi)}(t)\right) d t+\gamma e^{-\delta(\tau)} \ln X^{(\lambda, \pi)}(\tau)\right]
$$

with $\gamma \geq 0$ a constant.

By applying Theorem 3.3 to the case when the coefficients $\mu, \sigma$ and $\theta$ are given by (4.7), (4.8) and (4.9) for each given $\pi$. we obtain the following:

Theorem 4.3 Define $\hat{\lambda}(t)$ as in (3.9). Then $\hat{\lambda}(t)$ is an optimal relative consumption rate independent of the portfolio chosen, in the sense that

$$
J(\hat{\lambda}, \pi) \geq J(\lambda, \pi)
$$

for all $\lambda, \pi$ such that $(\hat{\lambda}, \pi) \in \mathcal{A}$ and $(\lambda . \pi) \in \mathcal{A}$. 
Corollary 4.4 Suppose there exists an optimal pair $\left(\lambda^{*}, \pi^{*}\right) \in \mathcal{A}$ for Problem 4.2. Then

$$
\lambda^{*}(t)=\hat{\lambda}(t) \quad \text { for all } t \geq 0,
$$

where $\hat{\lambda}(t)$ is given by (3.9).

Thus we see that the optimal consumption-portfolio problem splits into an optimal consumption problem (with solution $\lambda^{*}=\hat{\lambda}$ ) and then - by substituting $\lambda=\lambda^{*}$ into (4.6) - an optimal portfolio problem. For the solution of optimal portfolio problems for an insider with logarithmic utility and in this type of market we refer to [DMØP2].

\section{References}

[B] A. Bensoussan: Stochastic Control of Partially Observable Systems. Cambridge Univ. Press 1992.

[BEJM] C. Blanchet-Scalliet, N. El Karoui, M. Jeanblanc and L. Martinelli: Optimal investment and consumption decisions when time-horizon is uncertain. Manuscript 2002.

[BØ1] F. Biagini and B. Øksendal: A general stochastic calculus approach to insider trading. Preprint, Dept. of Math., Univ. of Oslo 17/2002. To appear in Applied Math. \& Optim.

[BØ2] F. Biagini and B. Øksendal: Minimal variance hedging for insider trading. Preprint, Dept. of Math., Univ. of Oslo 9/2004.

[BP] B. Bouchard and H. Pham: Wealth-path dependent utility maximization in incomplete markets. Finance \& Stochastics 8 (2004), 579-603.

[DMØP1] G. Di Nunno, T. Meyer-Brandis, B. Øksendal and F. Proske: Malliavin calculus and anticipative Itô formulae for Lévy processes. Preprint, Dept. of Math., Univ. of Oslo 16/2003. To appear in Infinite Dim. Anal. Quantum Probab.

[DMØP2] G. Di Nunno, T. Meyer-Brandis, B. Øksendal and F. Proske: Optimal portfolio for an insider in a market driven by Lévy processes. Preprint, Dept. of Math., Univ. of Oslo 36/2003. 
[EJ] N. El Karoui and M. Jeanblanc: Random horizon. Manuscript 2004.

[HØ] Y. Hu and B. Øksendal: Optimal smooth portfolio selection for an insider. Preprint, Dept. of Math., Univ. of Oslo 12/2003.

[I] K. Itô: Spectral type of the shift transformation of differential processes with stationary increments. Trans. Amer. math. Soc. 81 (1956), 253263.

[ØS] B. Øksendal and A. Sulem: Partial observation control in an anticipating environment. Preprint, Dept. of Math., Univ. of Oslo 31/2003.

[P] P. Protter: Stochastic Integration and Differential Equations. Second Edition 2004. Springer-Verlag.

[RV1] F. Russo and P. Vallois: Forward, backward and symmetric stochastic integration. Probab. Theory Rel. Fields 99 (1993), 403-421.

[RV2] F. Russo and P. Vallois: Stochastic calculus with respect to continuous finite quadratic variation processes. Stochastic and Stochastics Reports 70 (2000), 1-40.

[S] W. Schoutens: Lévy Processes in Finance. Wiley 2003. 